Swarthmore College

Works

\title{
Anthropomorphism In Sign Languages: A Look At Poetry And Storytelling With A Focus On British Sign Language
}

\author{
R. Sutton-Spence \\ Donna Jo Napoli \\ Swarthmore College,dnapoli1@swarthmore.edu
}

Follow this and additional works at: https://works.swarthmore.edu/fac-linguistics

Part of the Linguistics Commons

Let us know how access to these works benefits you

\section{Recommended Citation}

R. Sutton-Spence and Donna Jo Napoli. (2010). "Anthropomorphism In Sign Languages: A Look At Poetry And Storytelling With A Focus On British Sign Language". Sign Language Studies. Volume 10, Issue 4. 442-475. DOI: $10.1353 /$ sls. 0.0055

https://works.swarthmore.edu/fac-linguistics/26

This work is brought to you for free by Swarthmore College Libraries' Works. It has been accepted for inclusion in Linguistics Faculty Works by an authorized administrator of Works. For more information, please contact myworks@swarthmore.edu. 


\section{Anthropomorphism in Sign} Languages: A Look at Poetry and Storytelling with a Focus on British Sign Language

IN THIS ARTICLE we explore the use of anthropomorphism in telling a story or poem in a sign language and ask how it may be achieved. Although we base most of our findings on the works of Paul Scott and Richard Carter, leading British Deaf poets and storytellers, anthropomorphism, from ordinary conversation to poetry and storytelling, is common in many sign languages, so we draw freely on examples from other signers and other sign languages where necessary.

Anthropomorphism is common (even rampant) and occurs when we "ascribe human appearances and feelings to any animate or inanimate being." (Spada I997, 37). We might anthropomorphize because of an interest in animals, perhaps out of the (misguided) idea that doing so will help us understand animal behavior (Crist 2000), or out of a desire to help the audience gain a greater sense of connectedness to animals and nature in general (Moore 2008), or for some other reason. Alternatively, we might anthropomorphize for the very different reason that our nonhuman objects are really humans in disguise. This enables us to illuminate the experiences of humanity by projecting them onto objects that are free of characteristics that may cloud the analogous

Rachel Sutton-Spence is Reader in Deaf Studies at the University of Bristol. Donna Jo Napoli is Professor of Linguistics at Swarthmore College. 
human situation (Daston and Mitman 2005) or onto objects that have characteristics we want to explore (such as not using a spoken language). In this article we highlight how the use of anthropomorphism can create and portray Deaf perspectives on the world and, thus, contribute to the cohesion of Deaf communities.

Throughout our discussion our attention is on the linguistic methods used in sign anthropomorphism. We suggest that there is a cline or scale of anthropomorphism in signing, one that depends on a number of factors, including the skills and intention of the signer, the animacy of the entities represented, the form of their bodies, and the form of vocabulary signs referring to those entities. While anthropomorphism attributes human characteristics to nonhuman entities, animism attributes life to nonliving entities (such as a mountain, a train, or a piece of pastry) without necessarily giving them human attributes. However, skilled signers anthropomorphize the whole range of entities (from animate to inanimate) frequently and with apparent ease by embodying those entities and exploiting both manual and nonmanual articulators.

\section{The Extent of Anthropomorphism}

Some philosophers and scientists (especially behavioral scientists) frown upon our (often unconscious) tendency to anthropomorphize (see Kennedy I992). We humans think, feel, behave, and communicate in our own uniquely human way; we know little about the mental and emotional lives of the animals around us. We have no evidence that inanimate entities such as trees, mountains, or airplanes have thoughts, feelings, aspirations, and intentions. Furthermore, we know that nothing else- animate or inanimate - spontaneously uses our human languages or any form of communication that would qualify as language under most linguistic definitions (such as having words, a syntax, the ability to refer to objects not present, and so forth; see Anderson 2004). For careful realists, anthropomorphism is actually defined negatively as a "misattribution of properly human traits" (Guthrie 1997, 52). Nevertheless, anthropomorphism for most of us is not a mistake, as we now argue.

We can draw the gross but useful generalization that human languages reflect people's distinctions between things most like themselves 
and things more and more different from themselves, with animacy being a major factor. We refer to this loosely clustered set of distinctions as the animacy cline. Our human languages reflect the fact that we distinguish classes among the entities of the world, where, if the classes show distinctions in privilege with respect to grammatical phenomena, human beings are invariably in the most privileged class (witness the use of formal address in many European languages and the system of honorifics in Japanese). Yi (Tibeto-Burman) languages have numeral classifiers that select co-occurring nominals according to semantic groups, the most widespread of which is human (Bradley 200I). Japanese restricts to animates the subjects of passive sentences that have transitive counterparts (Sato 1982). Yuchi (a Native American isolate, now spoken mostly in northeastern Oklahoma) has noun classes that distinguish living things from nonliving things, and then living things of a different tribal community from members of the community, and also some members of the community from others based on a variety of criteria (Linn I997, 200I). Navajo distinguishes eight groups of nominals arranged into a status hierarchy by semantic characteristics. This "animacy hierarchy" is pertinent to word order possibilities and, in particular, to which noun phrases can serve as subject and object in the same sentence (Creamer 1974, 30). People (except newborns, but including lightning) are in the highest group, and animals are in successively lower groups as they decrease in size until we reach inanimates that move (such as wind), plants and inanimates that don't move, and, finally, abstractions.

Importantly, the distinctions of the animacy cline demonstrated in spoken languages can be validated via anthropomorphism in all of the language communities we know of. The only possible exception we've read about is Pirahã, a native language of Brazil, for which Everett (2005) reports that the people do not tell stories of any kind (though, see the [often quite critical] responses to Everett included in the 2005 publication). One might expect that in such a society anthropomorphism would be anathema. To the contrary, Everett (personal communication, email, February I4, 2009) tells us that the Pirahã people talk about the behavior of animals and inanimate objects, saying things such as "The monkey says I will hide from your arrows" or "The sky says I will rain now." Given that even a community with- 
out a story tradition uses anthropomorphism (and the most extreme form-in which language is attributed to nonhumans), we believe it is safe to say that people anthropomorphize to some degree regardless of their culture (see Moore 2008 for a discussion of the ubiquity of anthropomorphism in Western cultures). Indeed, in Europe and Japan it is common to find animals addressed in the formal and with honorifics in fairy tales or children's stories. In Navajo, nouns from groups other than the highest are "occasionally 'personified' in legendary writing or storytelling. In this case the personified noun is treated as having equal status with whatever category of nouns it is interacting with in the sentence" (Creamer 1974, 37). Further, in Tasmanian vernacular English, people are more likely to refer to nonhumans whose sex they do not know (or who are not sexed) with gendered pronouns if the referent is a large animal rather than a small one (such as an insect) and if the referent is a large plant (such as a tree) rather than a small one (Pawley I995a, I995b, 2002). Thus, the anthropomorphism reflected in the use of these pronouns is sensitive to the same sort of distinctions relevant to Navajo noun-group membership.

When a community encounters new concepts or objects, anthropomorphism enables people to talk about them using analogies to the known object of the human form. The Western Apache, for example, upon first encountering motor vehicles, used the human body and the body of a horse (hitherto their means of transport) as a metaphor to relate linguistically to cars. Thus, everything from the windscreen to the front bumper was the face, the front wheels were the hands and arms, and the rear wheels and tires were the feet. In this sort of structural metaphor, the language maintains a clear mapping of "cognitive topology" between the structure of cars and the purposes of their components on the one hand and the structure and purpose of human and animal body parts on the other (Palmer 1996). In spoken languages, words for human body parts may be used for these metaphors; in sign languages, this mapping may be achieved directly by using the body parts to mean those body parts.

In a spoken language such as English, when talking about the behavior of animals and inanimate objects, it is often possible to avoid anthropomorphic language by selecting the most abstract technical terms, but mostly we use everyday words such as want, decide, defend, 
and surrender. Indeed, Kennedy (1992) points out a range of ways in which anthropomorphism is unintended and, thus, often goes unrecognized in the work of researchers, particularly those looking at animal behavior. With signers, the situation is perforce more extreme. The use of anthropomorphism is unavoidable given the medium of the message - the signer's body. That is, sign languages represent referents using the body. Once signers embody the entity in the story or poem, their body becomes the entity's body and thus can communicate all of the emotions of the entity, just as it can communicate all of the emotions of the signers themselves.

Signers can make the analogies involved in anthropomorphism more directly and preserve the cognitive topology more literally than speakers can. That is, when referring to the forelimbs of an entity, a signer can use the arms; when referring to an animal's eyes, signers can use their own eyes, and so on. Dorothy Miles, a pioneer of poetry in ASL and BSL, wrote in 1976, "Using Ameslan [ASL], it's very easy to imitate animal characteristics and behavior. In turn, animal stories and poems are good for demonstrating Ameslan" (reproduced in Miles I998, 26).

However, skilled signers, particularly in creative language such as poetry and storytelling, can go far beyond the obvious analogies involved in mapping, for example, a cat body onto a human body; they can and do map from inanimate objects onto the human body. In other words, skilled signers in their anthropomorphizing can move down the animacy cline that so many spoken languages are sensitive to. Some mappings from inanimate objects onto the human body do not require too much imagination - such as with the Western Apache case of appropriating the words bigan (arms) and bikee (feet) for the front and rear wheels of a car. However, other mappings demand considerable creativity, as we show later, and it is these cases that are vastly more common in sign than in spoken language. It is the use of the nonmanuals - in particular, the eyes and other parts of the face- that allow these less obvious mappings.

In sum, human languages in general can exploit anthropomorphism, and signers can do so with great effectiveness for entities along the entire animacy cline. 
Reasons for Anthropomorphism

Our next question then is why people anthropomorphize and why Deaf communities, in particular, relish anthropomorphism, as evidenced in its widespread use in sign stories and poetry and the warm reception of storytellers and poets in Deaf communities. We have already mentioned one common situation that seems to encourage anthropomorphism: when we encounter a novel concept or entity. But many other, and more telling, situations exist as well.

Moore (2008) argues that anthropomorphism offers a way of seeing and understanding our surroundings. Humans in all societies see human characteristics in the world around them (Guthrie 1997): faces in clouds, hands in gnarled vegetables, and religious figures in any number of unlikely objects, where those religious figures are typically human in shape. We explain and understand the actions of nonhuman entities as though they are motivated by human intentions or as though they are evidence of the hand of a divinity (particularly in the case of natural disasters), which, again, is understood to be in human guise. Through the ages some people have believed that human and numinous (the terms being interchangeable to varying degrees) spirits exist in plants, water, wind, and other natural entities.

Metaphor can be used to create a new reality, so a novel metaphor by a signer or speaker is an invitation to a new understanding of the world (Brennan I990 and Wilcox 2000 discuss metaphor in sign languages in considerable and enlightening depth). Paths to new understanding through new realities may be seen in many different tropes and in any language, but they are especially powerful with anthropomorphism and in sign languages. Evans (2008) mentions the Czech proverb "Kolik jazyků znáš, tolikrát jsi člověkem," which he translates as "For each language you know, you are a new person." Bechter $(2008,62)$ claims that the Deaf cultural worldview is that "the world is made of deaf lives," so one objective of deaf narratives is to show deaf lives where others might not see them. The ASL poet Clayton Valli, in his poem "Deaf World" (in the DVD included with Lindgren, DeLuca, and Napoli 2008), points out that a great part of the world does not hear; he lists rocks, water, trees, mountains, clouds- 
natural entities that surround us. To a hearing-speaking person, the fact that a dog hears and makes noise but a tree does neither may make it easier to anthropomorphize the dog than the tree. To a signing Deaf person, that difference is irrelevant with regard to anthropomorphizability. More relevant, as we will see, is the physical form of the entity.

Bechter (2008) suggests that stories that anthropomorphize see and value the world differently. They narrate the worldview of entities that cannot otherwise speak for themselves. This parallels the experience of many Deaf people, who are unable to speak for themselves in a hearing world. Liberating the nonhuman entities to show their position in the world through sign language liberates all of us to a certain extent, but especially the audiences who can see the analogy to their own situations.

Guthrie (I997) has observed that the attribution of human characteristics to other entities in our world may be determined by focusing on what matters most to us, seeing the world as we wish to see it, or offering the most likely explanation for what we see. Accordingly, in sign anthropomorphism, signers focus on what matters most to a Deaf person. Many Deaf people have told us that they particularly enjoy stories that relate to their own experience, so it should come as no surprise that they present nonhuman entities as signing Deaf entities with a set of shared fundamental characteristics. Isolating these fundamental characteristics from anthropomorphized entities can reveal to us what signers consider the most important features of being Deaf. So anthropomorphism, ironically perhaps, can reveal a Deaf worldview rather than anything about how nonhuman entities behave. Anthropomorphic signing, even while delighting and entertaining, challenges our view of what it means to be human in this world, that is, what it means to be at the top of the animacy cline, at the top of the hierarchy of valued members of this world. More particularly, it challenges views of what it means to be a Deaf human in a world where other people are Deaf as well, placing them at the top of the hierarchy of value.

Signed stories with Deaf characters that view the world entirely from a Deaf perspective are critical in Deaf folklore, where promotion and maintenance of the Deaf identity and worldview are central 
(Bechter 2008; Bahan 2006; Smith and Sutton-Spence 2007). In many of the BSL stories with nonhuman characters that we have seen, human and nonhuman characters interact. While the nonhuman entities tend to be treated as Deaf and are anthropomorphized so that they may sign, it is rarer for them to be treated as hearing and anthropomorphized with human attributes as befits a hearing human. Hearing characters, instead, are for the most part limited to humans. In other words, in these stories being Deaf is the default case (the reverse of how stories are typically presented in hearing cultures). So, for example, in Paul Scott's poem "Tree" (2006), the tree is Deaf, but the man is hearing. The same is true in June Smith's rather different story "The Tree" (I998). In Richard Carter's tales of the "Jack-in-a-Box," the "Goldfish Companion," and the "Owl Interpreter," the Deaf human protagonist and the Deaf nonhuman character sign, but the other characters are human and hearing.

Despite the tendency for deafness to be the default in these stories, there are hearing characters - many of whom fall into one of two roles: villains and allies. Increasingly in BSL fairy tales, the villain is portrayed as hearing. This is the case for both human and nonhuman villains, for example, the wolf in stories such as "Little Red Riding Hood" and "The Three Little Pigs" is portrayed as hearing. Additionally, although far less commonly, nonhuman allies of Deaf people are hearing but can sign, as we will see in our discussion of Richard Carter's "Goldfish Companion" and "Owl Interpreter."

The fact that the anthropomorphism in a given story sheds light on humanity (rather than on nonhuman entities) may be implicit or explicit. Paul Scott's "Tree" tells of a tree that grows from a seedling and stoically encounters many challenges in its lifetime before a man comes to chop it down, and another seedling takes its place. Scott explains to us that his story is a metaphor for the Deaf community, which stoically meets life's many challenges. His image of the man felling the tree stands for the constant threat to the Deaf community from the hearing world, and the sprouting of the new seedling offers hope as the Deaf community resists and survives. Told entirely through the eyes of the tree (often literally, as the eyes are crucial articulators here), the story never mentions deafness. While clever and entertaining, it importantly serves to educate and enculturate as Deaf 
audiences recognize the symbolism without explicit acknowledgement of the anthropomorphism.

On the other hand, although Richard Carter's story of the goldfish companion also epitomizes the genre of anthropomorphic storytelling, it does so with unusually overt analogy that is useful to us here as it exposes the purpose of so many anthropomorphic stories in sign. In this story, a lonely Deaf man decides to get a goldfish as a companion. At the pet shop he finds one lonely fish in a corner of the tank, isolated from the other fish. The owner tells him the fish is Deaf, so he buys it. The man and the fish sign together, but they are still isolated within their own environments. The man understands that he needs a community to support him, and, also being gay, he realizes he needs the company of other gay men. Looking for love, he goes to a gay bar and meets a hearing man. He teaches him to sign, and they find some happiness. He realizes his fish also needs to be with its own kind, so he goes back to the pet shop and finds a hearing fish that would like to learn to sign with the Deaf fish, and the two fish find some happiness as well. However, the man and his hearing partner fight over misunderstandings. The two fish also fight over misunderstandings. When the man discusses his plight with another friend, matters seem to improve. He buys a third fish, and things get better in the fish bowl as well, but, alas, only temporarily. The man comes to understand that the third fish is making things worse, so he removes the third goldfish and gives it to his friend. The two remaining fish, Deaf and hearing, settle down and fall in love. Then the man realizes his own friend is likewise making things worse, so he ceases contact, works things out with his partner, and they, too, settle down and find real love. In time the man's partner dies, and the other fish is also found floating on the surface of the water. So the man and the goldfish are together again, each alone.

This story is within a strong Deaf story tradition of addressing ideas about deafness, sign language, community, and hearing people. It goes further, however, considering the pull of other communities, as a Deaf gay man finds love outside the Deaf world. As with another, perhaps better known story, Ben Bahan's ASL story "Bird of a Different Feather" (2007), the ending is not the "happily ever after" that one might hope for, yet there is a great deal in the story that is very funny indeed as it is told, and the entertainment comes primarily from 
the anthropomorphism of the fish and the comparison of his experience with the man's. It is a painful story of loneliness and loss, and without the anthropomorphism to add humor, it might be too sad to tell.

Stories and poems with anthropomorphism often evoke strong emotions, and this is a large part of their draw. Deaf people have told us repeatedly that they especially value the empathy generated in narrative, humor, drama, and poetry. The audience wants to feel a close affinity with the characters portrayed. In BSL, one sign for the concept expressed by the English word empathy may also be glossed as CHANGE-PLACES-WITH. Using anthropomorphism, signers are able to change places with the nonhuman characters as they embody them, inviting audiences to come closer still to the characters' experiences. When Richard Carter signs with his "fins" (as we discuss later), it's as though we see the fish itself telling us its story. We now consider the range of ways this change of places is effected in sign languages.

\section{Methods of Anthropomorphism in Sign Language}

Here we discuss how nonhumans are given human characteristics in sign. We consider four main factors: the linguistic base that allows such play, the ability of the nonmanuals to anthropomorphize even when the manual articulators are signing in an ordinary way, the range of possibilities for both manual and nonmanual articulators when the signer engages in (almost) complete embodiment of the nonhuman character, and how nonhumans are portrayed as communicating via sign language.

\section{Linguistic Base}

In order to understand how nonhuman entities are portrayed in sign languages - including the embodiment we've alluded to-it is crucial to understand the linguistic structures behind their representation. It is clear that many signs are visually motivated, and since Mandel's pioneering work in I977 we have understood that signers may either show the referent (in depicting signs) or become that referent (in substitutive signs). In a depicting sign, the signer may sketch the outline or surface of the referent or simply point to it. In substitutive signs, 
the signer may act out the referent or the hand itself may become the referent.

Linguists have also come to understand that the signs that form the central part of the vocabulary attribute general meaning and are used to identify referents so that the signer can "say without showing" (Risler 2007, 73). However, other signs are used with deliberate illustrative intent to, in effect, "say by showing." There are many different ways to deal with this dichotomy of signs, but it is widely recognized as an important distinction. The signs that have no deliberate illustrative intent are sometimes termed "frozen" (or established, standard, core, or vocabulary signs; see Taub 200I). The signs with illustrative intent have been termed highly iconic structures (HIS) by researchers led by Christian Cuxac (1985, I996, 2000) working on French Sign Language (but are also called productive, classifier, or polycomponential signs). Highly iconic structures are created when signers transfer their image of the real world directly into the visual, spatial, and kinetic domain of sign language and may include transfers of person (also termed role shift, characterization, or role playing) and transfers of form and size (see Risler 2007 and Sallandre 2007 for more detailed summaries of these terms). Anthropomorphic devices in creative signing make extensive use of both these transfer types.

In transfers of person, the signer as a narrator "disappears," yielding the stage to the entity by essentially becoming that entity, so that a signer's gestures correspond to the gestures of the character, who is both referred to and literally presented. To this end the signer may embody an entity; thus, the signer's hands are understood to mean the character's hands, the signer's eyes mean the character's eyes, and so on. If the hands act as though manipulating an object, perhaps holding a spoon and moving it in a stirring action, we understand that the character is manipulating that object, for example, stirring something with a spoon. If the hand waves, we understand that the character is waving. Sallandre (2007, I08) mentions that the entity that the signer "becomes" may be "any entity: human, animal or thing . . . a little boy, a horse, a tree and so on." She makes no further comment upon this extraordinary phenomenon, perhaps because the guiding principles for transfer appear the same for any type of entity, so that the 
wonder of the signer "disappearing" to become something other than human seems perfectly ordinary.

Transfers of form and size describe the entity through proforms made by different handshapes that reflect the general size or form of the entity, such as being flat, long and thin, thick, solid, or having legs. These handshapes may be moved and placed in signing space to enable signers to show where the referent was located or how it moved. Transfers of form have often been described in other literature as classifier handshapes, which may refer to the whole entity or to only parts of it. Unlike in transfers of person (where the signer's articulators are understood to be the articulators of the entity - the hand is the hand, the mouth is the mouth, and so on), in these signs the articulator is understood to refer to the entity. Thus, a single upright finger in many sign languages is understood by transfer of form to mean a whole person (not an entity holding up a single finger as it would in transfer of person), and a hand placed low down and a little away from the trunk is understood to mean a foot (not an entity placing its hand low down and away from the trunk).

These transfers permit the extraordinary wealth of anthropomorphic signing.

\section{Nonmanuals Doing the Job of Anthropomorphizing}

Signers can use their hands to show the body-part classifier of the entity through transfer of person, in which case there is no specific anthropomorphic intent behind the representation. However, even in this situation, we frequently see emotions attributed to the nonhuman entity through nonmanual elements (any part of the body other than the upper limbs). That is, signers may blend their representation of nonhuman entities so that the representation of the nonhuman attributes is made through the hands, whereas human attributions (that is, the anthropomorphism) are shown through the nonmanuals.

Sometimes the upper nonmanuals alone anthropomorphize the nonhuman entity, while the trunk does not get involved. Sallandre (2007) describes how LSF signer Nasreddine Chab uses widespread arms with the head to become pastry that has just been rolled out and is ready to go into a pie pan. Thus far in this transfer, there is nothing anthropomorphic. However, since the whole body is the pastry, the 
face is unavoidably included, and that is where the anthropomorphism occurs as the pastry shows its surprise on suddenly becoming a pie crust.

In Maria Gibson's prize-winning BSL haiku "Kettle," the signer's trunk and head become the kettle and are seen as a container for boiling water. The hands represent the boiling water and are clearly not part of the kettle. This might not appear to illustrate anthropomorphism, yet Maria Gibson's eye gaze and mouth position make it clear that we are seeing the experience through the eyes of a kettle (which stares blankly ahead as it focuses completely on its task of holding boiling water) (figure I from Gibson 2006).

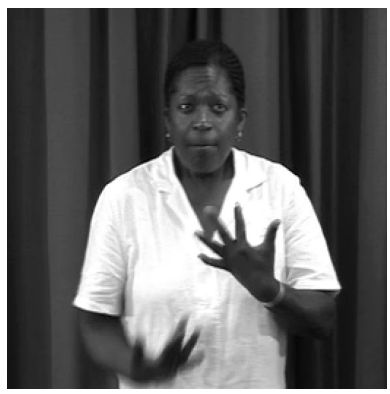

FIGURE I. Hands show water boiling while eyes and facial expression show the kettle's intense concentration (Maria Gibson).

In Paul Scott's poem "Tree," the seedling looks around furtively as it emerges from the soil. This effect is achieved by raising the hand representing the entity classifier of the seedling to face level, bringing the hand close to the eyes so that we understand that the eyes are those of the seedling (figure 2).

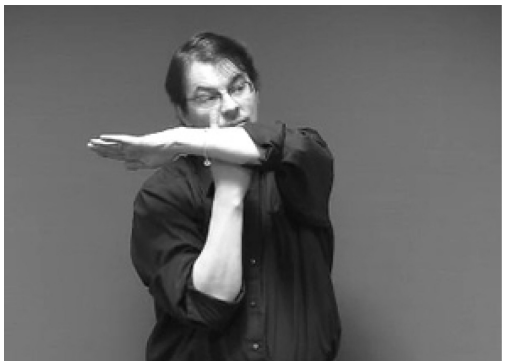

FIGURE 2. Seedling looks around cautiously as it emerges from the ground (Paul Scott). 
In Paul Scott's poem "Too Busy to Hug, Too Blind to See," the embodied sea looks up at the mountain, while at other points the embodied mountain looks both down on the sea and up to the sky as the rain and snow arrive.

In each anthropomorphized entity in these poems, the eyes communicate information about character, behavior, and emotions, so after a while we forget that pastry, kettles, trees, mountains, and the sea do not have eyes. The eyes play a major role in sign anthropomorphism despite the fact that many of the entities embodied do not have any. As Deaf entities, it is important that they should have eyes in order to perceive the world in a Deaf way (i.e., visually). (Sign language researchers, especially those working in LSF, are increasingly aware of the great importance of the eyes in the production of sign languages. See Risler 2007 and Sallandre 2007.)

Likewise, one's overall facial expression is a key part of sign anthropomorphism because it allows the embodied entity to communicate emotion or even grammatical information such as questioning. This is another important factor that contributes to an audience's ability to identify the entity as Deaf since an expressive face is part of Deaf communication in both affective and grammatical ways (McCullough, Emmorey, and Sereno 2005; Grossman and Kegl 2007). Facial expression is frequently exaggerated and may depict any human emotion.

Sallandre (2007) gives an example from LSF of a sign using transfer of form on the hands to represent a cat aggressively climbing a tree, plus facial expression clearly intended to be that of an aggressive cat. However, the face depicts human aggression, not feline. The signer does not flatten her ears or dilate her pupils because these options, available to a real feline, are not available using sign language articulators; rather, the representation of feline aggression has to be shown using the options available to a human signer.

In Paul Scott's (2003) BSL retelling of Aesop's fable of "The Hare and the Tortoise," the hare has a disdainful, overconfident facial expression, and the tortoise has a patient, determined one (figure 3).

Certainly we do not see disdain, overconfidence, patience, or determination on the faces of real hares and tortoises. Yet, because the signer uses his own face to portray these qualities and emotions, we accept them readily. 

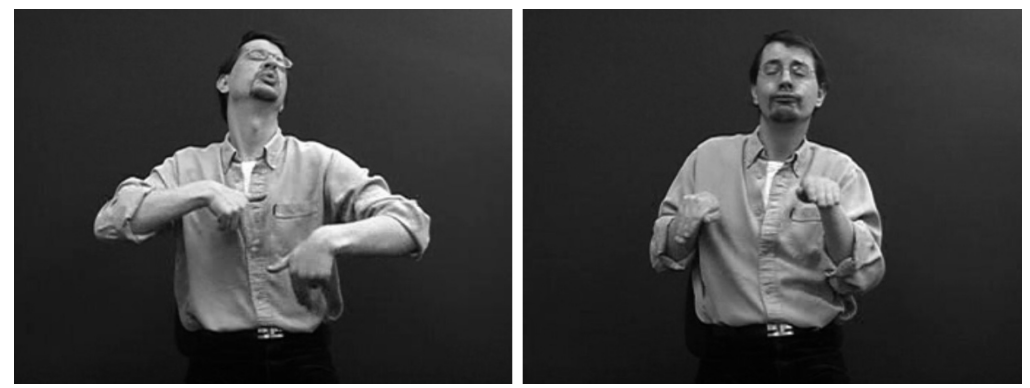

FIGURE 3. Human facial expressions for a disdainful hare and patient tortoise (note: manual articulators show animal body parts) (Paul Scott).

This is even possible in inanimate objects that have no face. Paul Scott's signed representations of a mountain and trees in "Too Busy to Hug, Too Blind to See" and "Tree," show their emotions of displeasure, pain, and annoyance in his face (see figures 4,5 , and 16 , for example).

While the upper nonmanuals alone can do the job of anthropomorphizing, often all of the nonmanual articulators (that is, including the trunk) work together to portray a character because they are all part of the transfer of person (that is, they all relate to a single character in a given action with a fixed perspective.)

In Paul Scott's “Too Busy to Hug, Too Blind to See," rain falls upon a mountain. The sign RAIN-FALLS uses both hands, while the rest of Scott's body represents the target of the rain: the mountain. If we think of the upright form of a human signer mapping onto the form of a mountain, the head is the summit, and the shoulders are the upper slopes. This in itself is not especially anthropomorphic even though it strongly links human shoulders to mountain slopes. However, the signer hunches his shoulders and ducks his head against the falling rain, adding the anthropomorphic dimension (figure 4).

Later in this poem both hands articulate the classifier of the mountain, but Scott's trunk suddenly flinches and twists, while the face shows pain and the mouth articulates "Ow!" (figure 5a). Whole-entity classifiers of something long and thin are then articulated against the trunk as trees start to shoot out of the mountainside (figure $5 \mathrm{~b}$ ). 


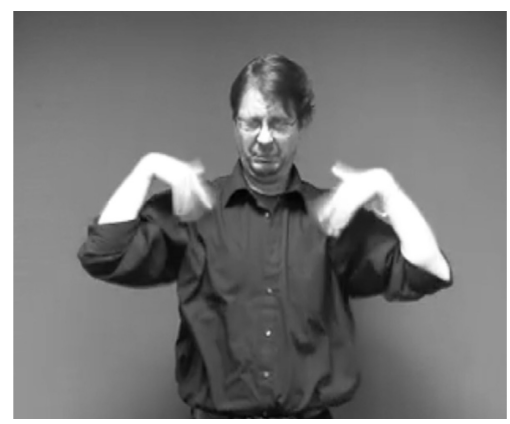

FIgURE 4. Rain falls on the mountainside. Hands articulating RAIN located on the shoulders as trunk, face, and head react in a human way (Paul Scott).
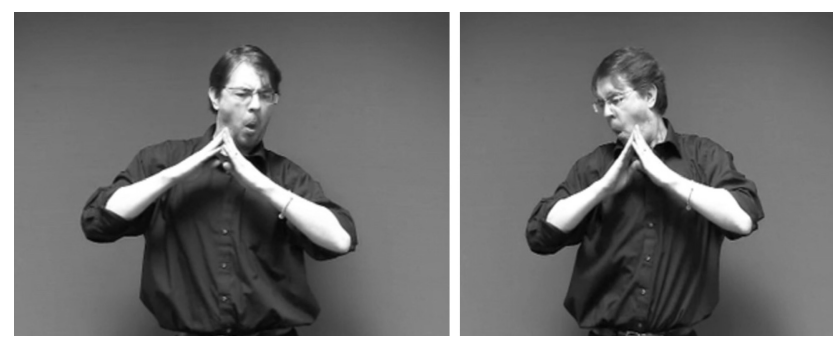

FIGURE 5 A. Hands show mountain slopes while the trunk flinches and the face grimaces to show the mountain in pain (Paul Scott).

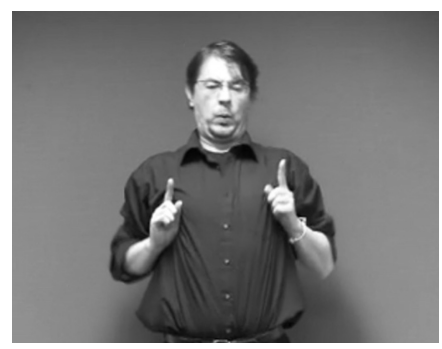

FIGURE 5 B. Hands show trees sprouting on the mountain (Paul Scott).

This particular example beautifully illustrates two aesthetic choices signers must make. First, they must decide what attributes of an entity they want to show, where the choice may be logical or absurd. Second, given those attributes, they must decide how to create a logic internal to the anthropomorphism (even in cases of the absurd). We 
readily believe that an animate being might feel pain if a growth erupted from its skin, but we do not expect an inanimate mountain to suffer when trees take root on its slopes. However, using the human body (here, excluding the arms) to represent a nonhuman allows us to accept the logic: Mountains feel pain when trees take root.

Later in this poem the sea tickles the mountainside, and the whole body twists, flinches, and wriggles in the mountain's clearly anthropomorphic response.

\section{Variations in Complete Embodiment}

By completely embodying a character, any nonhuman entity can be represented doing a range of activities. One choice for the signer in these cases is whether to have the nonhuman characters behave as if they have human form or as if they maintain their nonhuman form.

In Aesop's fable of "The Lion and the Mouse," told in any language, the lion is haughty, and the mouse is afraid and grateful and later slightly smug when it manages to free the lion from the hunters' net. In the BSL version by Carolyn Nabarro (2003) we see these human emotions on the face of the storyteller (figure 6), while the hands behave as human hands would in the acts of holding, fending off, and making a point.

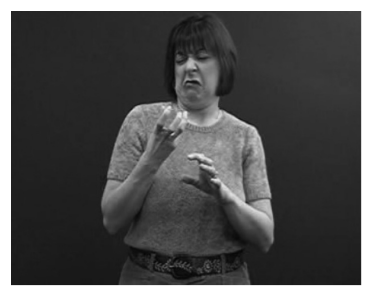

Lion looks at the mouse.

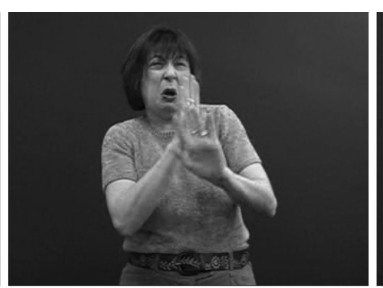

Mouse begs for mercy.

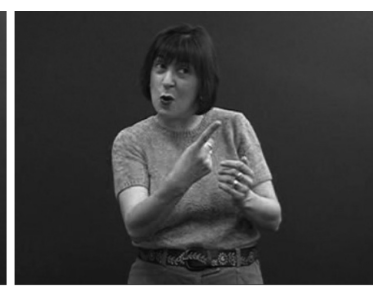

Mouse says, "I told you so."

FIGURE 6. Human manuals and facial expressions for a haughty lion and a frightened and slightly smug mouse (Carolyn Nabarro).

In Siobhan Donovan's BSL poem "Sixty-one Steps," old stone steps look up with compassion and concern for the elderly person walking upon them and, at the same time, reach up to hold that person (figure 7). The steps are given hands, which enable them to support the old 


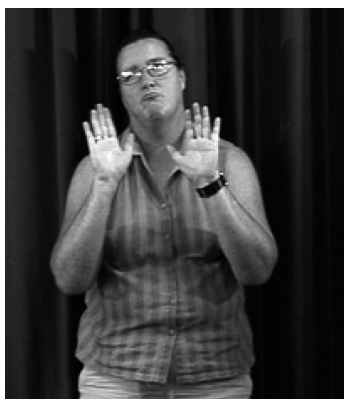

FIGURE 7. Facial expression to show stone steps looking up with compassion and human hands offering support (Siobhan Donovan).

person struggling up them. There is nothing about the physical form of a step that can be translated onto the human hands, but they are shown here in order to emphasize the assistance.

In this poem the eyes always look upward when the signer embodies the steps. We understand from this that the core being of a step is situated under the surface: The steps under our feet look up at the soles of our shoes.

Two different stories containing bird characters portray the birds as though they have human hands. In Philip Green's BSL version of "The Ugly Duckling," the mother duck settles down on her nest to knit while she waits for her eggs to hatch, and she knits just as a human would - as though she has human hands. In Ben Bahan's (2007) classic ASL fable, "Bird of a Different Feather," Father Eagle holds and reads the sports pages of the newspaper just as a human might - again as though he has human hands (figure 8).

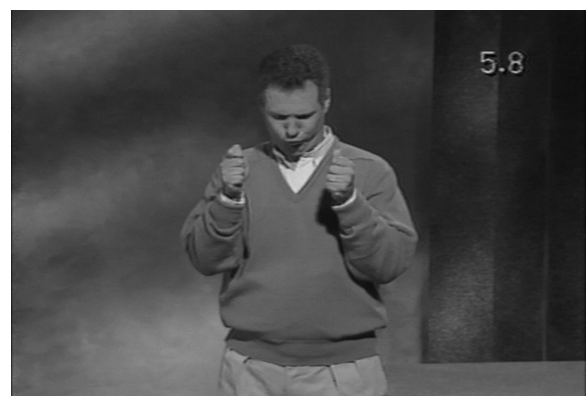

FigURE 8. Father Eagle reading the newspaper with human hands (Ben Bahan). 
In these last two examples, audiences have just been shown indisputably birdlike characteristics but then accept this human behavior attributed to the birds since they see the eagle reading.

Alternatively, in the same two works, the activities of nonhuman characters may be performed using body-part classifiers. We are shown that the mother duck in "The Ugly Duckling" gathers her eggs around her by scooping them up with her wing, not a human-shaped hand. In Dorothy Miles's BSL performance of the same story the hens snigger at the ugly duckling behind their wings. The arm portrays the hen's wing in a nonanthropomorphic way, but lifting it to cover the face while sniggering is an anthropomorphic action (figure 9).

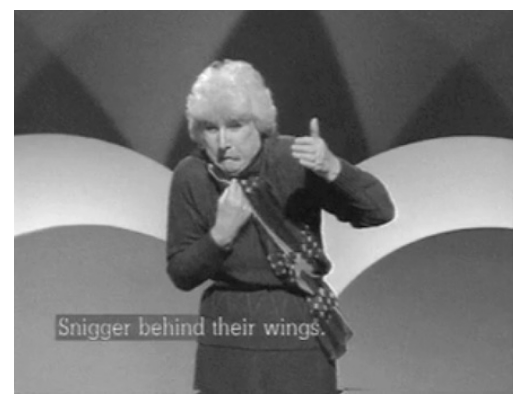

FIGURE 9. The arm as a wing to show a hen sniggering behind its wing (Dorothy Miles).

This method of using classifiers to represent an object and at the same time to perform the action that that object might perform is highly complex conceptually, yet visually transparent. The following examples demonstrate that transparency.

In June Smith's BSL poem "The Tree" (I998), the branches of the trees are shown by using her arms, while her fingers make the twigs, and when the trees see (and feel) the woodsman approaching, they perform the human action of holding hands with these nonhuman body parts.

In Guy Bouchauveau's comic story about a biplane (I994; told using international signs), the wings of the plane are shown using simple body-part classifiers. Yet they engage in human behavior as they rub each other to stay warm, scratch itches, feel their way through dense clouds, and hit each other during a squabble. 
The ice-covered mountain in Paul Scott's "Too Busy to Hug, Too Blind to See" wipes its brow in the heat of the summer sun. We see a straightforward representation of a human hand wiping a human brow and a human facial expression of suffering in the heat. Mountains have no physical equivalent of hands and faces, but signers briefly personify them to permit this. However, the nondominant hand remains to show one mountainside, making it very clear that the mountain is sweating, not a person. Notice that the examples in the previous section exploited the fact that the nonmanuals can be independent articulators, while here the fact that each hand can be an independent articulator is exploited, with one hand forming part of a sign (for MOUNTAIN) and the other engaging with the rest of the body to form the completely different sign WIPE-SWEAT (see Miller I994 for discussion of this kind of simultaneity in ordinary conversational contexts and Dudis 2004 for a discussion of body partitioning) (figure Io).

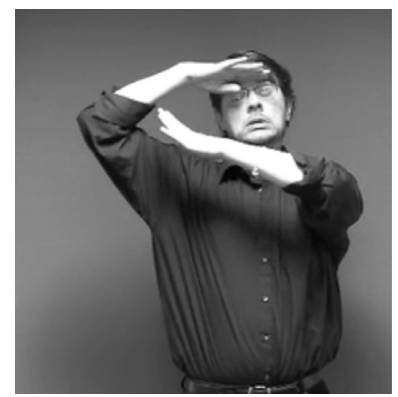

FIGURE I O. Left hand indicates the mountain slope; right hand signs WIPE-SWEATFROM-BROW (Paul Scott).

Another masterful technique is to use a sign that indicates an object whose form is not humanlike in any way and reanalyze it in an anthropomorphic context as a new sign that indicates hands. For example, the form of the sea itself does not have anything that might physically map onto human hands, but the form of the BSL sign SEA does. In Paul Scott's “Too Busy to Hug, Too Blind to See" the waves are represented with a fully open 5 handshape, entirely free of any sense other than water. A signer looking at SEA or WAVES would not see the articulators as indicating hands but as some sort of collective 
entity classifier to show the large, undulating surface of the sea (see, e.g., Brennan 1990 and Johnston and Schembri 2007 for more about surface classifiers). In much of the poem, this is how the hands are correctly interpreted, but when the signer anthropomorphizes the sea, the articulating hands may be interpreted as the hands of the sea. The signer's hands show the waves as they tap the mountain to get its attention, tickle it, and stroke it —all human actions performed by a hand that morphs steadily back and forth between representing a collective entity classifier and an embodied hand (figure I I).

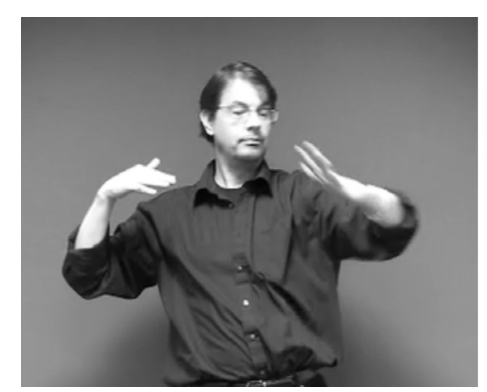

The sea

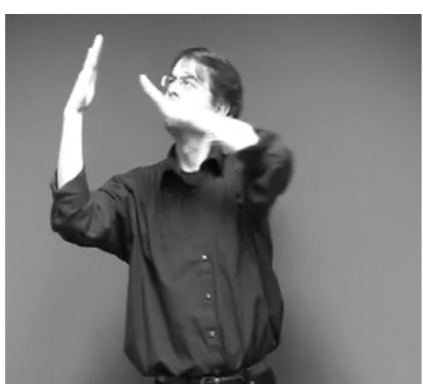

The sea taps the mountain

FIGURE I I. Left hand representing the sea, first as the sea and then as a hand tapping the mountain (Paul Scott).

Later in this poem we find another example of the same sort of morphing, this time involving the mountain. As the ice and snow return to the mountain in winter, the mountain shivers with cold just as a human would. The signer's cheeks puff out against the cold, and his teeth chatter while the sign MOUNTAIN is held on the hands. Even while maintaining this sign, however, the hands shake and shiver, giving the impression simultaneously of the whole mountainside shivering and also the mountain's hands shaking with cold. Perhaps there is even a hint that the hands can also be seen as a body-part classifier for chattering teeth.

Notably, in all of the examples in this section, the entities do not use their hands to sign-only to be (exist) or to act. Now we turn to examples in which the hands are used to sign, as well as examples in which the nonmanuals narrate the story. 


\section{Nonhuman Entities Communicating Visually}

Attributing language to nonhuman entities is arguably the most extreme example of anthropomorphism, yet it is remarkably common. In spoken languages the anthropomorphized entities are usually allowed to speak as humans speak (allowing for changes in pitch, volume, and speed). In sign languages the anthropomorphized entities rarely speak and instead use visual communication-both manual and nonmanual—since they are presented from the Deaf worldview.

\section{Signing}

It is usually a given in a sign narrative that the nonhuman entities understand sign language (which, as we noted earlier, necessitates that they can see-by embodying inanimate objects, signers lend them their own eyes).

In a full personification of the entity, we are invited to accept the entity as human, and it can then sign in the ordinary way. When Thomas the Tank Engine talks to a tractor, the Three Little Pigs defy the Wolf, or the Hare and the Tortoise challenge each other to a race, all may be clearly identified as nonhuman in the description and representation of their other behavior and movements, but when they sign, they sign in an unexceptionally human way. That is the logic of the absurdity that we alluded to earlier: Once we accept that these entities are sufficiently human to use our language, we accept that they have hands with which to sign.

In Paula Garfield's retelling of “The Gingerbread Man” in BSL, the gingerbread man is repeatedly identified as having solid, fingerless hands, using a closed B handshape. However, when he signs, he signs exactly as a human would, using the full range of handshapes. The fox that finally eats the gingerbread man uses his paws (signed with closed A handshapes) to perform many human activities such as wiping his lips after his meal, but when he signs, he uses fully human signs.

Alternatively, the signer can work within the confines of the particular entity being anthropomorphized as the signer represents that entity signing. In that case, the challenge is to supply appropriate signing articulators. Signing is most easily represented where there are close analogies between the physical bodies that the storyteller 
can exploit, particularly when the nonhuman entities have hands and fingers that move. For example, to tell a story about a monkey, the signer's fingers move almost identically to those of the monkey. In Richard Carter's tale of the (Deaf) Jack-in-a-Box who teaches the little (Deaf) boy about temptation, self-discipline, remorse, and forgiveness, the Jack-in-a-Box is definitely not human (he sways on his spring and holds his arms out to the side), but he has hands that are sufficiently humanlike to sign, as in signing "Shh" (figure I2).

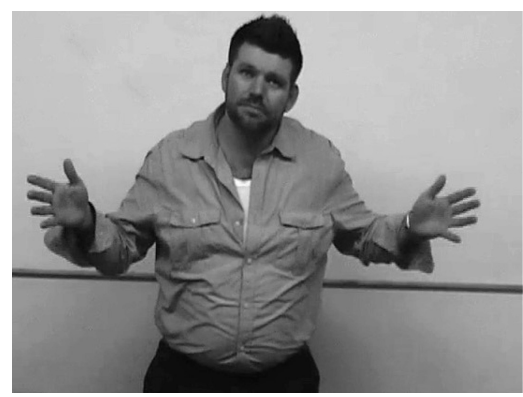

Jack-in-a-Box

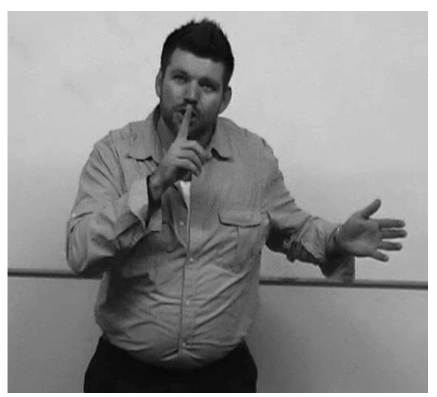

Jack-in-a-Box says, "Shh!"

FIGURE I 2. Signer's hands represent a nonhuman entity with hands (Richard Carter).

Further along this scale are analogies with other nonhumans that do not have humanlike hands and fingers. However, our basic human anatomy is frequently sufficiently similar to that of other animals that we can utilize isomorphic (or at least homomorphic) body parts to show signing that is notably not human and more related to the character of the entity. Bears, birds, and fish have limbs that are not shaped quite like a hand, but they can flex and move, so they can be modified sufficiently to allow them to sign. Some creatures (animals with hooves, for example) have less potential for articulation similar to finger articulation, so representing their signing challenges a signer's skill and ingenuity.

When a nonhuman is portrayed signing, it is possible to employ the handshape of the body-part classifier representing the limb while retaining the other parameters of movement, location, and orientation for whatever sign is being made. Richard Carter's story titled "Birthday" features a bear that signs to a small child. Each sign is made with 
the clawed, spread 5 handshape, as though signing with bear paws. The bear can even fingerspell using these paws (figure I3).

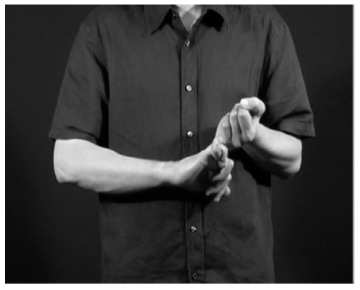

S

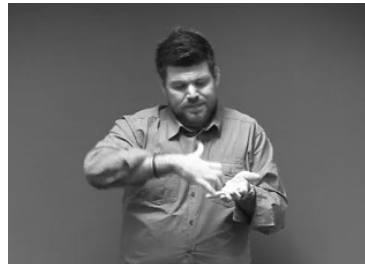

S

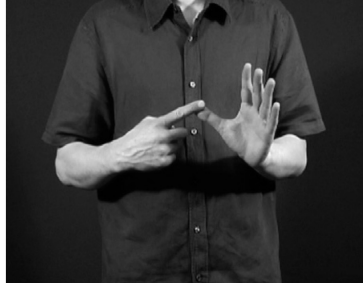

A

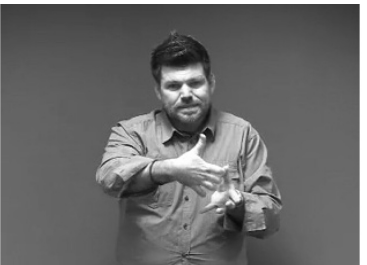

A

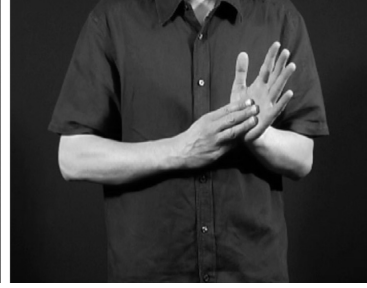

M

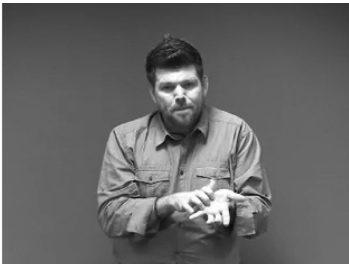

M

FIGURE I 3 . Fingerspelling "Sam" conventionally in BSL and using bear claws (Richard Carter).

(This is possible in the two-handed BSL manual alphabet, where the exact finger configuration is less crucial than it is in ASL. The relative arrangement of the two hands and their movements carry enough information for BSL fingerspelling to be interpretable). In a charming twist to this tale, the bear turns out to be the child's father dressed in a bear costume. Thus, we have a human signer portraying a human signer portraying a signing bear.

Signing animals occur frequently in Richard Carter's performances. In his tale "Owl Interpreter," the owl uses its wings to sign. The body-part classifier representing the wings uses the $\mathrm{B}$ handshape. Thus, all of the signs use the B handshape, although they also use the other expected parameters for the signs. When the owl says to the group of Deaf school children, SHH ME INTERPRET ALL HELP-YOU SHE TEACHER DON'T-KNOW SHH ("Hush, don't tell. I'll interpret for you all and help you, and your teacher won't know. Hush."), the hand- 
shape never alters from the $\mathrm{B}$ that represents the shape of the owl's wings. In BSL these signs would ordinarily use the $1, \mathrm{~V}$, and $\AA$ handshapes, but the message is fully comprehensible with the $\mathrm{B}$ handshape because the movement, orientation, and location are conventional (figure I4).

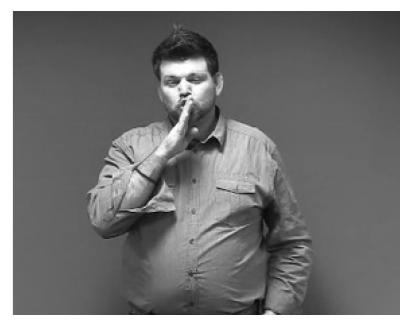

HUSH

(usually 1 handshape)

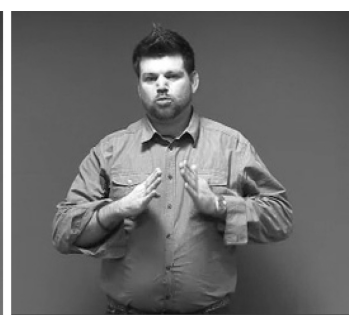

INTERPRET

(usually V handshape)

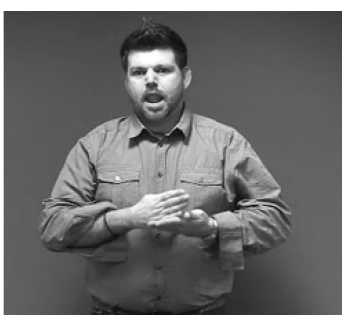

HELP

(USUALLY A HANDSHAPE)

FIGURE I 4. Owl signing by maintaining correct location and movement while using a handshape for an owl's wing (Richard Carter).

Richard Carter's ingenious solution for having a reindeer sign makes use of the antlers. Although the antlers are fixed to the head and cannot flex and move through space the way that the animal's legs could, they are - crucially — shaped like a hand (not in reality, of course, but in the BSL sign DEER). For the sake of the story, the antlers are allowed to flex so the reindeer can sign (for a more detailed description of his story titled "Snowglobe," featuring the signing reindeer, see SuttonSpence and Napoli 2009). Although the antlers are permitted to flex so that the handshape may vary, Richard suspends the logic of the absurd at that point and fixes the location of all of the signs at the temples (where the antlers are). In his "Goldfish Companion," the same device is seen: The goldfish signs with its fins. As the fins are located on the chest, all of the signing takes place there. The handshape and movement of the signs are the conventional ones, but the signs are all anchored to the chest.

Generally we may expect that it is easier to represent the signing of animate beings than of inanimate ones because of our shared evolutionary anatomy. Trees, however, appear to be a special case because some sort of accidental homomorphism is still possible. The form of 
the human body maps so well onto the form of the tree that signing trees are very popular in stories and poems.

Inanimates that can move may be next in the cline. These include mechanical things like airplanes and elevators or forces of nature like wind and water, which may also be given human attributes but are less likely to communicate through sign language because they lack physical analogues for hands. They tend to communicate through nonmanual channels, but opportunities exist for representing other anthropomorphic characteristics through their movement. Master storytellers, however, might yet find a way to enable these to sign. Guy Bouchauveau, for example, uses the airplane's wings to sign (see Bouchauveau I994; Sutton-Spence and Napoli 2009).

Other inanimates do not move by themselves. Some have to be thrown or rolled, such as a ball or an apple, and others are unlikely ever to move, such as a mountain. Still, it is possible to allow even these entities to have hands (or at least arms) through full personification, so that they may engage in human activities, although they rarely sign.

In fact, in an incident many years ago (the incident, in fact, that first introduced one of us to the delights of signed humor), someone did just that. A group of people were sitting under an apple tree in summer when an apple fell and landed on someone's head. The joker of the group immediately took on the role of one of the other apples on the tree, looked around furtively, and gave a hard, sharp nudge with his elbow to knock the neighboring apple off the branch to fall onto the people below. The signer's whole body took on the role of the apple. With the furtive eye gaze and firm-set facial expression of an apple bent on mischief, there was no complaint that an apple has no elbows with which to nudge.

Novice BSL storytellers often either personify an entity totally, so that they simply sign as though the entity were human, or keep to entities with forms sufficiently similar to the human form to allow a simple embodiment. More skilled signers can move along the animacy cline, but the most accomplished storytellers ignore it altogether. They can anthropomorphize a wall, perhaps one that a heavy person is leaning against or one that is about to get hit with a hammer and nail. Paul Scott clearly shows us the world from the perspective of a tree as it interacts with humans and animals - the cat that climbs it, the dog that 
cocks his leg against it, the blind man who feels his way around it, and the man who comes to chop it down. In "Sixty-one Steps" Siobhan Donovan shows the feelings and behavior of a set of old stone steps as people tramp up and down them.

\section{Nonmanual Narration}

When an entity does not have a physical attribute whose shape can be mapped onto arms or hands or when the signer chooses not to represent them, the signer can still represent communication (beyond just emotions-discussed earlier) and narration by recruiting other body parts, including the trunk and shoulders but particularly the head. Peters (200I, I 40) refers to such narratives as "face stories."

The head can be used to represent spherical objects. By embodying them, the signer allows them to have facial expressions and eyes with which to communicate and perceive the world in a Deaf way (such as the pinball in Bahan 2006). Manual signing is not represented because the entities are understood not to have hands. The signer's skill then is in revealing not only the entities' feelings and intentions but also their actions, reactions, and, important for us here, attempts at communication or narration entirely nonmanually. Peters describes one example of a signer who becomes an ice cube that is put into a glass, covered in soda, floats, is taken into a person's mouth, and is spat back into the glass, all shown entirely through the movement of the head and facial parts.

Bechter (2008) observes that these entities might be considered disempowered because of their inability to communicate easily with the world or control what is happening to them, which is analogous to the frequent experience of Deaf communities. He describes these face stories as subaltern because the protagonists cannot speak or sign (because they have no hands) and cannot control their destinies (again because they have no hands), and yet they have feelings and manage to tell us stories.

In Nasreddine Chab's recipe for an apple pie, the signer becomes an apple, but only the head takes on this transfer. His head dips as the apple winces under the chopping knife that slices it to go into the pie (Sallandre 2007). We can assume that the apple is disempowered and at the mercy of outside forces because, if we accept that the apple has human characteristics, we also assume that-like us - it would not wish to be chopped up and put in a pie. Importantly, as Sallandre observes, 
the signing during this anthropomorphization of the apple is not entirely nonmanual. Chab does produce manual signs, but they refer to the knife that cuts the apple- the signs do not show the apple's hands. As Bechter claims, these handless entities are subject to the whims and powers of the hands around them.

Another tactic is to use the head and face to show emotions, actions, and reactions, while the hands, via classifiers, show what the entity is. Throughout John Wilson's performances of the BSL haiku "Lift," (as an elevator is termed in British English) his hands simply articulate the elevator doors, which frame what we understand to be the essence of the elevator behind the doors. The core of the elevator's "body" (and its attributed mind and personality) is shown on the face. In one performance of this poem the elevator is merely an object that observes: It looks around in eager anticipation of receiving its passengers and grows increasingly dejected when none appear. In another performance of this poem, the elevator attempts to communicate visually with potential passengers despite having no hands for the purpose, looking at — and smiling encouragingly at — different people in turn and motioning with the head for them to come inside.

It might go without saying that the mouth is not used for the entity to speak in these examples. These nonhumans are all Deaf, and even though they don't sign, their communication is strictly visual. The mouth may be used to mean a mouth for an inanimate entity, but it is not employed for speech. For example, in Paul Scott's "Too Busy to Hug, Too Blind to See," the wind uses its mouth to blow on the sea in order to drive water into clouds so it will fall as rain onto the mountain, but it does not speak (figure I 5 ).

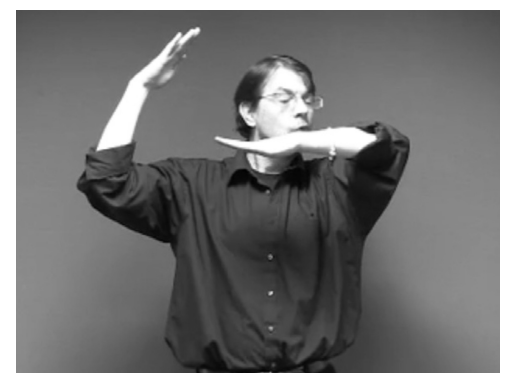

FIGURE I 5 . "Wind blows on the sea." Left hand shows the sea, right hand shows the mountain, and head and mouth show anthropomorphized wind blowing (Paul Scott). 
To sign BLOW in BSL in relation to a human blowing, we would pucker up the mouth as if blowing. Wind, however, does not blow in this way (because it does not have a mouth), and BSL WIND-BLOW is very different from HUMAN-BLOw-but Paul Scott has anthropomorphized the wind.

In Paul Scott's "Tree," the tree shows emotions with its mouthgrimacing in horror when it sees the axe approaching and twisting in pain as the axe cuts deep-but it does not speak. The mouth is not used for speaking because these are Deaf entities, communicating in other, visual, ways.

That said, the mouth may be used to articulate the visual mouth pattern of words in the contact spoken language, in keeping with normal Deaf behavior in many signing communities. Although the extent of the use of these mouthings varies in different sign languages, it is well attested in BSL (see, e.g., Sutton-Spence and Day 2000) and other languages. In "Too Busy to Hug, Too Blind to See," the waves wash with increasing strength and height up the mountainside until the mountain turns to the sea and mouths crossly "What?!" (figure I6).

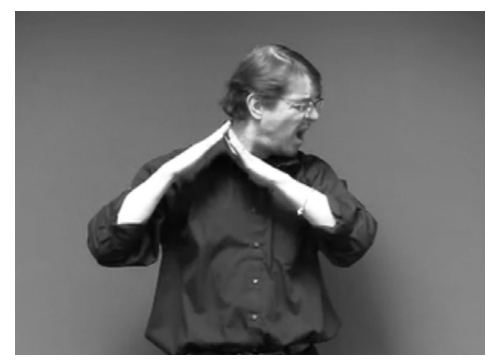

Figure i 6. Mountain mouths, "What?!" to the sea below, using the mouth while the hands show the mountain.

As an example of anthropomorphism this episode is a gem, showing the anthropomorphic characteristics of both sea and mountain as they communicate in a Deaf way. The sea taps the mountain to get its attention in a way that is socially acceptable in Deaf culture. The entity classifier WAVES-WASH-UP-THE-MOUNTAINSIDE uses an open 5 hand. In the context of washing against the mountain, however, it can morph into a real hand tapping the mountain. The 
mountain, clearly familiar with Deaf social rules of attention getting, has been ignoring the sea until the waves become too insistent. Both hands are in use to represent the two sides of the mountain, so the mouth is used, together with the very irritated facial expression of the mountain, to say "What?!"

\section{Conclusion}

Anthropomorphism in sign language demonstrates the extraordinary potential for storytellers and poets to present alternative worldviews through the direct transfer of person of nonhuman entities. With an apparent ease and a striking linguistic economy that belie the conceptual complexity of what they are doing, skilled signers animate the inanimate (no matter how far down the animacy cline) and anthropomorphize the nonhuman. We have described how human behavior and emotions may be attributed to these entities through the use of manual and nonmanual features, especially the eyes. In the hands (and faces) of skilled Deaf storytellers, any kind of entity can behave as Deaf, engaging in culturally appropriate Deaf behavior and creating empathy with Deaf audiences. The entities are permitted to communicate visually, especially by signing. The absurd but careful logic of the anthropomorphism makes this visual technique amusing, while still allowing it to be taken as a serious comment on and/or insight into the Deaf worldview.

\section{Acknowledgments}

We would like to thank Paul Scott and Richard Carter for their kind permission to use their work. Their performances were funded by the University of Bristol Faculty of Social Sciences and Law Research Development Fund. The illustrations in figures 3 and 6 are taken from stories collected by the ECHO sign language project (http://www .let.ru.nl/sign-lang/echo). The illustrations in figures 2,7 , and Io are taken from the BSL haiku website (http://www.bslhaiku.co.uk/ poems.html). Figure 8 is from the DVD of Bird of a Different Feather \& For a Decent Living by Ben Bahan and Sam Supalla (1994/2007). We thank Ben Bahan and DawnSignPress for their generous permission to 
use this. We also thank Don Read for his kind permission to use the illustration of Dorothy Miles in figure 9. Tim Northam modeled our conventional manual letters in figure $\mathrm{I} 4$, and Dan Berlin gave us media help, and we hereby thank both of them. Finally, we thank the anonymous reviewer whose insightful criticisms, so kindly delivered, helped to reshape the organization of this final draft.

\section{References}

Anderson, S. 2004. Dr. Doolittle's Delusion: Animals and the Uniqueness of Human Language. New Haven: Yale University Press.

Bahan, B. 2006. Face-to-face Tradition in the American Deaf Community: Dynamics of the Teller, the Tale, and the Audience. In Signing the Body Poetic: Essays on American Sign Language Literature, ed. H.-D. L. Bauman, J. L. Nelson, and H. M. Rose, 2 I-50. Berkeley: University of California Press.

— Series, Teacher's Set DVD. San Diego: DawnSignPress.

Bechter, F. 2008. The Deaf Convert Culture and Its Lessons for Deaf Theory. In Open Your Eyes: Deaf Studies Talking, ed. H.-D. L. Bauman, 60-82. Minneapolis: University of Minnesota Press.

Bouchauveau, G. 1994. Deaf Humor and Culture. In The Deaf Way: Perspectives from the International Conference on Deaf Culture, ed. C. Erting, R. Johnson, D. Smith, and B. Snider, 24-30. Washington, D.C.: Gallaudet University Press.

Bradley, D. 200 I. Counting the Family: Family Group Classifiers in Yi (Tibeto-Burman) Languages. Anthropological Linguistics 43(I): I-I 7.

Brennan, M. 1990. Word Formation in British Sign Language. Stockholm: University of Stockholm Press.

Creamer, M. H. I974. Ranking in Navajo Nouns. Diné Bizaad Náníl'h. [Navajo Language Review] I: 29-38.

Crist, E. 2000. Images of Animals: Anthropomorphism and Animal Mind. Philadelphia: Temple University Press.

Cuxac, C. I985. Esquisse d'une typologie des langues des signes. In Autour de la langue des signes: Journée d'études Io, ed. C. Cuxac, 35-60. Paris, Université René Descartes.

- I996. Fonctions et structures de l'iconicité des langues des signes. $\mathrm{PhD}$ diss., Université Paris V.

- 2000. La Langue des signes française (LSF): Les voies de l'iconicité. Faits de Langues, n. I 5-I6. Paris: Ophrys.

Daston, L., and G. Mitman, eds. 2005. Thinking with Animals: New Perspectives on Anthropomorphism. New York: Columbia University Press. 
Donovan, S. 2006. Sixty-one Steps. http://www.bslhaiku.co.uk/poet_so.html (accessed January 2009).

Dudis, P. 2004. Body Partitioning and Real-space Blends. Cognitive Linguistics I 5(2): $223-38$.

Evans, N. 2008. Grammar and Psychosocial Cognition: What Linguistic Diversity Can Tell Us about Social Cognition. http://www-I.unipv.it/ wwwling/Evans.pavia.soccog.talk.pdf (accessed January 23, 2009).

Everett, D. L. 2005. Cultural Constraints on Grammar and Cognition in Pirahã. Current Anthropology 46(4): 62 I-646.

Gibson, M. 2006. Kettle. http://www.bslhaiku.co.uk/poet_mg.html (accessed January 2009).

Grossman, R., and J. Kegl. 2007. Moving Faces: Categorization of Dynamic Facial Expressions in American Sign Language by Deaf and Hearing Participants. Journal of Nonverbal Behavior 3 I (I): 23-38.

Guthrie, S. E. I997. Anthropomorphism: A Definition and a Theory. In Anthropomorphism, Anecdotes, and Animals, ed. R. W. Mitchell, N. S. Thompson, and H. L. Miles, 50-58. Albany: State University of New York Press.

Johnston, T., and A. Schembri. 2007. Australian Sign Language (Auslan): An Introduction to Sign Language Linguistics. Cambridge: Cambridge University Press.

Kennedy, J. S. I992. The New Anthropomorphism. Cambridge: Cambridge University Press.

Lindgren, K., D. DeLuca, and D. J. Napoli, eds. 2008. Signs and Voices: Deaf Culture, Identity, Language, and Arts. Washington, D.C.: Gallaudet University Press.

Linn, M. S. I997. Yuchi and non-Yuchi: A Living Classification. Florida Anthropologist 50(4): I 89-96.

- 200I. A Grammar of Euchee. PhD diss., University of Kansas.

Mandel, M. I977. Iconic Devices in American Sign Language. In On the Other Hand: New Perspectives on American Sign Language, ed. L. A. Friedman, 57-I07. New York: Academic Press.

McCullough S., K. Emmorey, and M. Sereno. 2005. Neural Organization for Recognition of Grammatical and Emotional Facial Expressions in Deaf ASL Signers and Hearing Nonsigners. Cognitive Brain Research 22(2): I93-203.

Miles, D. 1998. Bright Memory: The Poetry of Dorothy Miles, ed. D. Read. Feltham, Middlesex: British Deaf History Society.

Miller, C. I994. Simultaneous Constructions in Quebec Sign Language. In Word-order Issues in Sign Language: Working Papers, ed. M. Brennan and G. H. Turner, 89-II2. Durham: International Sign Linguistics Association.

Moore, B. 2008. Ecology and Literature: Ecocentric Personification from Antiquity to the Twenty-first Century. New York: Palgrave Macmillan. 
Nabarro, C. 2003. The Lion and the Mouse. http://www.let.ru.nl/sign-lang/ echo (accessed June 2008).

Palmer, G. I996. Toward a Theory of Cultural Linguistics. Austin: University of Texas Press.

Pawley, A. I995a. Gender Assignment in Tasmanian English. Typescript. Department of Linguistics, Research School of Pacific and Asian Studies, Australian National University.

- I995b. Some Characteristics of Tasmanian Vernacular English. Typescript. Paper read to Australian Linguistic Society Conference, Canberra, September I995.

- 2002. Using He and She for Inanimate Referents in English: Questions of Grammar and World View. In Ethnosyntax: Explorations in Grammar and Culture, ed. N. J. Enfield, I I I-37. New York: Oxford University Press.

Peters, C. 200 I. Rathskeller: Some Oral-traditional and Not-so-traditional Characteristics of ASL Literature. In Deaf World: A Historical Reader and Primary Sourcebook, ed. L. Bragg, I29-46. New York: New York University Press.

Risler, A. 2007. A Cognitive Linguistic View of Simultaneity in Process Signs in French Sign Language. In Simultaneity in Signed Languages, ed. M. Vermeerbergem, L. Leeson, and O. Crasborn, 73-Ior. Amsterdam: Benjamins.

Sallandre, M.-A. 2007. Simultaneity in LSF Discourse. In Simultaneity in Signed Languages, ed. M. Vermeerbergem, L. Leeson, and O. Crasborn, I03-26. Amsterdam: Benjamins.

Sato, C. I982. Some Properties of Inanimate Subject Passives. Papers in Japanese Linguistics 8: 177-90.

Scott, P. 2003. The Hare and the Tortoise. http://www.let.ru.nl/sign-lang/ echo (accessed June 2008).

- 2006. The Tree. Paul Scott's Poetry DVD. Coleford, U.K.: Forest Books.

Smith, J. I998. The Tree. In video accompanying The Linguistics of British Sign Language: An Introduction, by R. Sutton-Spence and B. Woll. Cambridge: Cambridge University Press.

Smith, J. and R. Sutton-Spence. 2007. What Is the Deaflore of the British Deaf Community? Deaf Worlds 23: 44-69.

Spada, E. I997. Amorphism, Mechanomorphism, and Anthropomorphism. In Anthropomorphism, Anecdotes, and Animals, ed. R. W. Mitchell, N. S. Thompson, and H. L. Miles, 37-49. Albany: State University of New York Press.

Sutton-Spence, R., and L. Day. 2000. Mouthings and Mouth Gestures in British Sign Language. In The Hands Are the Head of the Mouth: The Mouth 
Anthropomorphism in Sign Languages | 475

as Articulator in Sign Languages, ed. P. Boyes-Braem and R. SuttonSpence, 69-85. Hamburg: Signum.

Sutton-Spence, R., and D. J. Napoli. 2009. Sign Language Humor: The Linguistic Underpinnings. Dublin: Trinity College Dublin Press.

Taub, S. 200 I. Language from the Body: Iconicity and Metaphor in American Sign Language. Cambridge: Cambridge University Press.

Wilcox, P. 2000. Metaphor in American Sign Language. Washington, D.C.: Gallaudet University Press. 